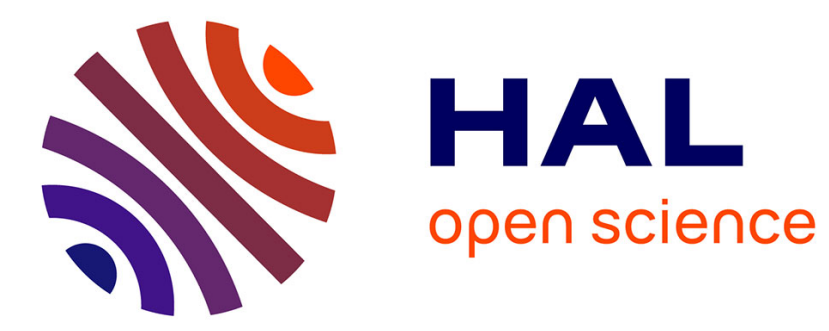

\title{
De Bruijn and Kautz Bus networks
}

Jean-Claude Bermond, Robin Dawes, Fahir Ö. Ergincan

\section{To cite this version:}

Jean-Claude Bermond, Robin Dawes, Fahir Ö. Ergincan. De Bruijn and Kautz Bus networks. Networks, 1997, 30 (3), pp.205-218. 10.1002/(SICI)1097-0037(199710)30:33.0.CO;2-P . hal-03210219

\section{HAL Id: hal-03210219 \\ https://hal.inria.fr/hal-03210219}

Submitted on 27 Apr 2021

HAL is a multi-disciplinary open access archive for the deposit and dissemination of scientific research documents, whether they are published or not. The documents may come from teaching and research institutions in France or abroad, or from public or private research centers.
L'archive ouverte pluridisciplinaire HAL, est destinée au dépôt et à la diffusion de documents scientifiques de niveau recherche, publiés ou non, émanant des établissements d'enseignement et de recherche français ou étrangers, des laboratoires publics ou privés. 


\title{
De Bruijn and Kautz Bus Networks
}

\author{
Jean-Claude Bermond, ${ }^{1}$ Robin W. Dawes, ${ }^{2}$ Fahir Ö. Ergincan ${ }^{2}$ \\ ${ }^{1}$ SLOOP, Joint Project 13S-CNRS/UNSAINRIA, 2004 Route des Lucioles, BP 93, F-06902 \\ Sophia-Antipolis, FRANCE \\ ${ }^{2}$ Nortel Technology, P.O. Box 3511, Station C, Ottawa, Ontario, K1Y 4H7 Canada
}

\begin{abstract}
Our aim was to find bus interconnection networks which connect as many processors as possible, for given upper bounds on the number of connections per processor, the number of processors per bus, and the network diameter. Point-to-point networks are a special case of bus networks in which every bus connects only two processors. In this case, de Bruijn and Kautz networks and their generalizations are known to be among the best families of networks with respect to the aforementioned criteria. In this paper, we present the directed de Bruijn bus networks, which connect two or more processors on a bus and contain the point-to-point de Bruijn networks and their generalization as a special case. We study two different schemes of the directed de Bruijn bus networks. We also show that the directed Kautz bus networks can be defined in the same manner.
\end{abstract}

\section{INTRODUCTION}

A bus interconnection network is a collection of processing elements (processors) and communication elements (buses). The processors produce and/or consume messages and the buses provide communication channels to exchange messages among the processors. Every bus provides a communication link between two or more processors.

For practical reasons, a processor may be connected only to a limited number of buses (this number is known as the processor degree) and a bus may connect only a limited number of processors (this number is known as the bus size). Therefore, messages may have to be relayed

Contract grant sponsors: French GDR-PRC C ${ }^{3}$; Natural Science and Engineering Research Council of Canada; School of Graduate Studies and Research, Queen's University at Kingston by a number of intermediate processors before arriving at their destinations, and thus the message transmission time becomes a function of the distance (measured in terms of the number of buses traversed by a message) between processors. The maximum distance over all pairs of processors is the network diameter.

For given upper bounds on the processor degree, bus size, and network diameter, the construction of bus networks with a maximal number of processors is an important problem in the design of interconnection networks. Other design parametérs such as network reliability, ease of message routing, and balanced message traffic throughout the network should also be taken into consideration.

In the case of traditional point-to-point networks, where a link can connect only two processors, the aforementioned problem has been extensively studied in the literature. As a result, several families of networks with large number of processors for a given degree and diame- 
ter have been proposed. (Surveys on this topic can be found in $[1,2,6,8]$.) Among them, de Bruijn and Kautz networks appear to have many other desirable properties (for details, see $[7,17]$ ). The classical definition of the de Bruijn networks is based on alphabets. These networks can be generalized for any number of processors by using arithmetic congruences (see $[13,16]$ ).

When the bus size is taken into account as an extra parameter, the problem becomes more complicated. There are relatively few results in the literature on this subject (for a survey, see [5]). The problem has been studied for a small degree and a small diameter where the tools from the Design Theory and Hypergraph Theory are useful. Although there are a few direct methods to construct bus networks, most of the bus networks proposed in the literature are obtained, in fact, from the known pointto-point networks using some transformation. A simple transformation is to partition the set of links into subsets. Each subset of links defines a new bus. Doty [9] used this idea to construct bus networks from the undirected de Bruijn graphs.

In this paper, we present and study some construction schemes that generalize the point-to-point de Bruijn and Kautz networks to bus networks. Since the de Bruijn and Kautz networks are modeled by directed graphs (digraphs), the schemes proposed in this paper impose an orientation on the buses, i.e., certain processors on a bus can use it only for sending messages, while others can use it only for receiving. Therefore, we use directed hypergraphs to model these bus networks.

This paper is organized as follows: In Section 2, we introduce our notation and terminology and define the problem formally. In Section 3, we propose two schemes to obtain bus networks from the de Bruijn digraphs. The networks of Scheme 1 are constructed using alphabets (Section 3.3). Although they can be defined and generalized arithmetically (see Section 3.7), this is not always practical. In Section 3.4, we introduce the networks of Scheme 2 by using arithmetical congruences. They can also be defined using alphabets, but, in contrast with the first scheme, their alphabetical definition is complicated (Section 3.5). We show in Section 3.6 that the two schemes, in general, give nonisomorphic networks. In Section 4, we study the Kautz bus networks.

\section{DIRECTED HYPERGRAPHS}

A directed hypergraph $H$ is a pair $\left(\vartheta^{\circ}(H), \mathcal{E}(H)\right)$, where $\vartheta(H)$ is a nonempty set of elements (called vertices) and $\mathcal{E}(H)$ is a set of ordered pairs of nonempty subsets of $\vartheta(H)$ (called hyperarcs). If $E=\left(E^{-}, E^{+}\right)$is a hyperarc in $\varepsilon(H)$, then the nonempty vertex sets $E^{-}$and $E^{+}$are called the in-set and the out-set of the hyperarc $E$, respectively. The sets $E^{-}$and $E^{+}$need not be disjoint.
The hyperarc $E$ is said to join the vertices of $E^{-}$to the vertices of $E^{+}$. Furthermore, the vertices of $E^{-}$are said to be incident to the hyperarc $E$ and the vertices of $E^{+}$are said to be incident from $E$. The vertices of $E^{-}$are adjacent to the vertices of $E^{+}$, and the vertices of $E^{+}$are adjacent from the vertices of $E^{-}$.

If $E$ is a hyperarc in a directed hypergraph $H$, then $\left|E^{-}\right|$is the in-size and $\left|E^{+}\right|$is the out-size of $E$ where the vertical bars denote the cardinalities of the sets. The maximum in-size and the maximum out-size of $H$ are

$$
s^{-}(H)=\max _{E \in \delta(H)}\left|E^{-}\right| \text {and } s^{+}(H)=\max _{E \in \delta(H)}\left|E^{+}\right|,
$$

respectively. The order of $H$ is the number of vertices in $\eta^{\circ}(H)$ and is denoted by $n(H)$. The number of hyperarcs in $H$ is denoted by $m(H)$. We note that a digraph is a directed hypergraph $G=(\vartheta(G), \mathscr{E}(G))$ with $s^{-}(G)$ $=s^{+}(G)=1$.

Let $v$ be a vertex of $H$. The in-degree of $v$ is the number of hyperarcs that contain $v$ in their out-set and is denoted by $d_{H}^{-}(v)$. Similarly, the out-degree of vertex $v$ is the number of hyperarcs that contain $v$ in their in-set and is denoted by $d_{H}^{+}(v)$. [ When $H$ is clear from the context, we will write simply $d^{-}(v)$ and $d^{+}(v)$, respectively.] The maximum in-degree and the maximum out-degree of $H$ are, respectively,

$$
d^{-}(H)=\max _{v \in \tau^{(}(H)} d_{H}^{-}(v) \text { and } d^{+}(H)=\max _{v \in \tau^{\tau}(H)} d_{H}^{+}(v)
$$

A walk in $H$ from vertex $u$ to vertex $v$ is an alternating sequence of vertices and hyperarcs $u=v_{0}, E_{1}, v_{1}, E_{2}, v_{2}$, $\ldots, E_{k}, v_{k}=v$ such that vertex $v_{i-1} \in E_{i}^{-}$and $v_{i} \in E_{i}^{+}$ for each $1 \leq i \leq k$. The length of the walk is equal to the number of hyperarcs on it. The distance from vertex $u$ to vertex $v, d_{H}(u, v)$, is the length of a shortest walk from $u$ to $v$. The diameter of the hypergraph, $D(H)$, is the maximum of the distances between pairs of vertices in the hypergraph:

$$
D(H)=\max _{u, v \in v(H)} d_{H}(u, v)
$$

If $H$ is a directed hypergraph, its dual $H^{*}$ is found as follows: Every hyperarc $E$ in $H$ is represented by a vertex $e$ in $H^{*}$, and every vertex $v$ in $H$ is represented by a hyperarc $V$ in $H^{*}$. The incidence relation between vertices and hyperarcs in $H^{*}$ is as follows: Let $V^{-}$and $V^{+}$be the in-set and out-set of hyperarc $V$ in $H^{*}$. Vertex $e$ of $H^{*}$ is in $V^{-}$if and only if $v \in E^{+}$in $H$. Similarly, $e$ is in $V^{+}$if and only if $v \in E^{-}$in $H$.

We will call a directed hypergraph with maximum outdegree $d$, diameter $D$, and maximum out-size $s$ a $(d, D$, $s)$-directed hypergraph. [Note that one may define $(d$, $D, s)$-directed hypergraphs in different fashions. For ex- 
ample, one may assume that $d^{-}(v)+d^{+}(v) \leq d$, for every vertex $v$ and/or $\left|E^{-}\right|+\left|E^{+}\right| \leq s$ for every hyper$\operatorname{arc} E$. We will not consider these cases in this article.] Let $n(d, D, s)$ denote the maximum number of vertices over all $(d, D, s)$-directed hypergraphs. It is easy to show that

$$
n(d, D, s) \leq \sum_{i=0}^{D}(d s)^{i} .
$$

This bound is known as the Moore bound for directed hypergraphs, and the directed hypergraphs that attain this bound are called the directed Moore hypergraphs. Ergincan and Gregory [10] showed that directed Moore hypergraphs do not exist if $d s>1$ or $D>1$. The $(d, D, s)$ directed hypergraph problem consists of finding $(d, D$, $s$ )-directed hypergraphs with a maximum number of vertices or finding large $(d, D, s)$-directed hypergraphs.

The drawing of directed hypergraphs can be very complex. Therefore, it is useful to represent a directed hypergraph $H$ with a bipartite digraph,

$$
R(H)=\left(\vartheta_{1}(R) \cup \vartheta_{2}(R), \&(R)\right),
$$

called the bipartite representation digraph of $H$. Every vertex $v$ in $\vartheta(H)$ is represented by a vertex $v$ in $\vartheta_{1}(R)$ and every hyperarc $E$ in $\mathcal{E}(H)$ is represented by a vertex $e$ in $\eta_{2}(R)$. We draw an arc from $v \in \eta_{1}(R)$ to $e$ $\in \vartheta_{2}(R)$ if and only if $v \in E^{-}$in $H$. Similarly, we draw an arc from $e \in \vartheta_{2}(R)$ to $v \in \vartheta_{1}(R)$ if and only if $v$ $\in E^{+}$in $H$.

If $H$ is a $(d, D, s)$-hypergraph and $R(H)$ is its bipartite representation digraph, then the maximum out-degrees in $\eta \%_{1}(R)$ and in $\vartheta_{2}(R)$ are $d$ and $s$, respectively. The distance between two vertices of $\vartheta_{1}(R)$ is at most $2 D$, but the diameter of $R(H)$ can be $2 D, 2 D+1$, or $2 D+2$ as the vertices of $\vartheta_{1}(R)$ and $\vartheta_{2}(R)$ do not play the same role. So, the $(d, D, s)$-directed hypergraph problem is partly related to but different from the $\left(d_{1}, d_{2} ; D^{\prime}\right)$-bipartite digraph problem, i.e., finding large bipartite digraphs with maximum vertex out-degrees $d_{1}$ and $d_{2}$ and diameter $D^{\prime}$. This problem was considered for $d_{1}=d_{2}$ by Fiol and Yebra [11].

If only the adjacency relations between the vertices in a directed hypergraph $H$ are considered, we can use the underlying multidigraph $\hat{H}$ [also called associated multidigraph and denoted by $A(H)]$. The vertex set of $\hat{H}$ is the same as that of $H$. There are as many arcs from $u$ to $v$ in $\hat{H}$, as there are hyperarcs $E$ in $H$ such that $u \in E^{-}$ and $v \in E^{+}$. Then, a hyperarc of $H$ corresponds to a "complete bipartite digraph" (shortly diclique), and a directed hypergraph corresponds to a multidigraph with a partitioning of its arc set into dicliques.

\section{DE BRUIJN BUS NETWORKS}

\subsection{De Bruijn Digraphs}

An important class of digraphs with a large number of vertices and small diameter is the de Bruijn digraphs. One of the ways to define de Bruijn digraphs is based on alphabets. For given integers $d \geq 2$ and $D \geq 1$, vertices of the de Bruijn digraph $B(d, D)$ are labeled with words of length $D$ from an alphabet $A$ of size $d$. Let $\left(a_{1}, a_{2}\right.$, $\left.\ldots, a_{D}\right)$ be the label of a vertex $v$ of $B(d, D)$. Then, $v$ is adjacent to the vertices with labels $\left(a_{2}, \ldots, a_{D}, \alpha\right)$ for all $\alpha \in A$. It is easy to see that $B(d, D)$ has $d^{D}$ vertices, diameter $D$, and in- and out-degree $d$. Asymptotically (for fixed $D$ ), de Bruijn digraphs have about the maximum number of vertices.

Reddy et al. [16] and Imase and Itoh [13] independently proposed a family of digraphs that can be defined on any number of vertices. The vertices of these digraphs are numbered with integers modulo $n$ ( $n$ is the number of vertices), and an arc is drawn from vertex $v$ to vertex $u$ if $u \equiv d v+\alpha(\bmod n)$, where $0 \leq \alpha \leq d-1$. The diameter of these digraphs is $\left\lceil\log _{d} n\right\rceil$, and if $n=d^{D}$, the resulting digraph is isomorphic to $B(d, D)$. We call these digraphs the generalized de Bruijn digraphs and denote them by $G B(d, n)$.

Another way to define the de Bruijn digraphs is by using the line digraph iterations [12]. If $G$ is a digraph, its line digraph $L(G)$ is found as follows: Every arc $E$ in $G$ is represented by a vertex $e$ in $L(G)$; vertex $e$ is adjacent to vertex $f$ in $L(G)$ if and only if the arc $E$ is incident to the arc $F$ in $G$. The line digraph of $B(d, D)$ is $B(d$, $D+1) \cdot[B(d, 1)$ is a complete digraph on $d$ vertices with self-loops.]

\subsection{General Remarks}

In the following sections, we will present two schemes to construct directed de Bruijn hypergraphs. In both schemes, the underlying multidigraph is a (generalized) de Bruijn digraph of out-degree $d s$ where $d$ and $s$ are positive integers. Therefore, ${ }_{,}$our schemes correspond to a partition of the arc set of the (generalized) de Bruijn digraphs into dicliques. This fact was pointed out to us by J. Bond (private communication) who showed that for $n=(d s)^{D}$ the proposed partitioning schemes are refinements of the partitioning of the de Bruijn digraph into "natural dicliques," i.e., the arcs joining the vertices ( $\alpha$, $\left.a_{1}, \ldots, a_{D-1}\right)$ to the vertices $\left(a_{1}, \ldots, a_{D-1}, \alpha\right)$, for all $\alpha \in A$. (The reader familiar with the de Bruijn digraphs will notice that this partition follows from the line digraph property. The arcs incident to and from a vertex yield a diclique in the line digraph.) A similar idea was used by Doty [9] to construct undirected hypergraphs.

Scheme 1 is based on alphabets (Section 3.3). It can 
be generalized for arbitrary number of vertices (but with some parity restrictions on the number of hyperarcs and out-size) using arithmetical congruences (Section 3.7). However, the arithmetical definition is somewhat clumsy. On the other hand, Scheme 2 is best defined using arithmetical congruences for an arbitrary number of vertices (Section 3.4). An alphabetical definition of this scheme is also possible (Section 3.5), but it is not as simple as that of Scheme 1. Scheme 2 has the additional property that its dual hypergraph also belongs to the same family.

In the alphabetical definitions, we will use the following notation to define the composition of the labels of the vertices and the hyperarcs: Let $k$ be a nonnegative integer and let $A$ and $B$ be two alphabets of sizes $d$ and $s$, respectively. $[A]^{k}$ denotes any string of $k$ letters from $A$. $[A B]^{k}$ denotes any string of $2 k$ letters $(a, b, \ldots, a, b)$, where $a \in A$ and $b \in B$. The string is null if $k=0$.

\subsection{De Bruijn Bus Networks-Scheme 1}

The vertex labels of this scheme are of the form $[B A]^{D}$, and the hyperarc labels are of the form $[A][B A]^{D-1}[A]$. If $E$ is a hyperarc with label $\left(a_{0}, b_{1}, a_{1}, \ldots, b_{D-1}, a_{D-1}\right.$, $\left.a_{D}\right)$, it joins the set of vertices $E^{-}=\left\{\left(\beta, a_{0}, b_{1}, a_{1}, \ldots\right.\right.$, $\left.\left.b_{D-1}, a_{D-1}\right) \mid \beta \in B\right\}$, to the set of vertices $E^{+}=\left\{\left(b_{1}\right.\right.$, $\left.\left.a_{1}, \ldots, b_{D-1}, a_{D-1}, \beta, a_{D}\right) \mid \beta \in B\right\}$. We repeat the incidence rules by taking a vertex as our reference point: The vertex

$$
\left(b_{1}, a_{1}, b_{2}, a_{2}, \ldots, b_{D-1}, a_{D-1}, b_{D}, a_{D}\right)
$$

is incident to the set of hyperarcs

$$
\left\{\left(a_{1}, b_{2}, a_{2}, \ldots, a_{D-1}, b_{D}, a_{D}, \alpha\right) \mid \alpha \in A\right\}
$$

and is incident from the set of hyperarcs

$$
\left\{\left(\alpha, b_{1}, a_{1}, \ldots, a_{D-2}, b_{D-1}, a_{D-1}, a_{D}\right) \mid \alpha \in A\right\} .
$$

In other words, the set of hyperarcs that a vertex is incident to is found by shifting the vertex label to the left by one, disposing of $b_{1}$, and introducing a new letter from alphabet $A$ from the right end. Alternatively, the set of hyperarcs that a vertex is incident from is found by shifting all letters (except $a_{D}$ ) to the right by one (disposing of $b_{D}$ ) and adding a new letter from alphabet $A$ from the left end.

We denote these hypergraphs by $B_{1}(d, D, s)$. Observe that if $s=1$ the letters $b_{i}$ can be eliminated from both vertex and hyperarc labels. In other words, the hypergraph $B_{1}(d, D, 1)$ is nothing more than the de Bruijn digraph
$B(d, D)$. Figure 1 depicts the bipartite digraph representation of $B_{1}(2,2,3)$.

Clearly, $B_{1}(d, D, s)$ has $(d s)^{D}$ vertices and $d^{2}(d s)^{D-1}$ hyperarcs. It is regular $\left[d^{+}(v)=d^{-}(v)=d\right.$, for every vertex $v]$ and uniform $\left(\left|E^{-}\right|=\left|E^{+}\right|=s\right.$, for every hyperarc $E$ ). Its diameter is $D$, since starting from any vertex we can reach any other vertex by at most $2 D$ shift operations, which, in turn, corresponds to visiting at most $D$ hyperarcs. Then, $n(d, D, s) \geq(d s)^{D}$ and, therefore, the number of vertices in this scheme approaches the Moore bound.

The underlying multidigraph of $B_{1}(d, D, s)$ is nothing more than the de Bruijn digraph constructed on an alphabet of size $d s$, the letters being pairs $(\beta, \alpha)$ with $\beta \in B$ and $\alpha \in A$. The "natural dicliques" defined in Section 3.2 are of the following form: They contain the arcs joining the vertices $\left(\beta, \alpha, b_{1}, a_{1}, \ldots, b_{D-1}, a_{D-1}\right)$ to $\left(b_{1}, a_{1}\right.$, $\left.\ldots, b_{D-1}, a_{D-1}, \beta^{\prime}, \alpha^{\prime}\right)$. Such a diclique can itself be partitioned into subdicliques by fixing $\alpha=a_{0}$ and $\alpha^{\prime}$ $=a_{D}$. This smaller diclique corresponds to the hyperarc $E=\left(a_{0}, b_{1}, a_{1}, \ldots, b_{D-1}, a_{D-1}, a_{D}\right)$.

\subsection{De Bruijn Bus Networks-Scheme 2}

In this section, we define a family of directed hypergraphs using arithmetic congruences. Let $n$ be the number of vertices and $d$ be the vertex out-degree. For reasons which will become clear later, we will assume that the number of hyperarcs $m$ and the hyperarc out-size $s$ satisfy the following conditions:

$$
\begin{aligned}
d n \equiv 0 & (\bmod m) \\
s m \equiv 0 & (\bmod n) .
\end{aligned}
$$

Assume that the vertices are numbered with integers modulo $n$ and that the hyperarcs are numbered with integers modulo $m$. The incidence rules are as follows: Vertex $v$ is incident to the hyperarcs

$$
E \equiv d v+\alpha \quad(\bmod m) \text { for all } 0 \leq \alpha \leq d-1,
$$

and the out-set of the hyperarc $E$ consists of the vertices

$$
u \equiv s E+\beta \quad(\bmod n) \quad \text { for all } \quad 0 \leq \beta \leq s-1 .
$$

Let $G B_{2}(d, n, s, m)$ dendte the hypergraphs constructed using the above rules. Note that if $s=1$ we obtain the generalized de Bruijn digraphs. Note also that Condition (1) is necessary to represent the vertices with integers congruent modulo $n$. If $v$ and $v+n$ are two integers (representing the same vertex), then they must be in the in-sets of the same hyperarcs, namely, we require that 


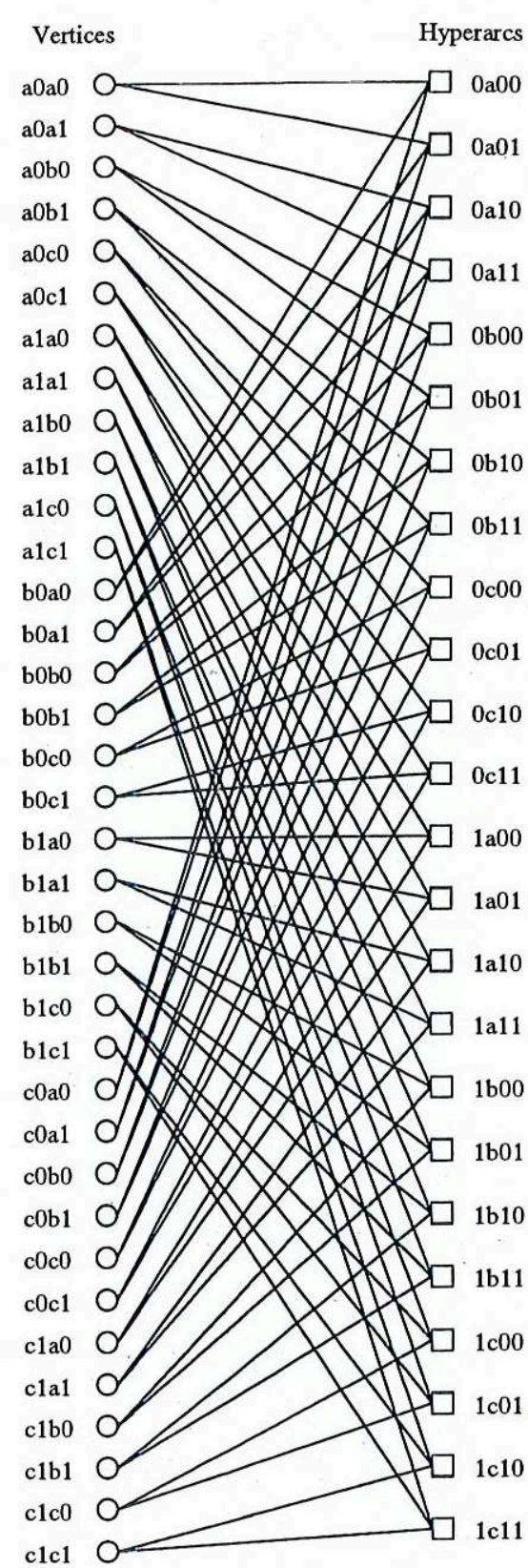

Connections from vertices to hyperarcs.

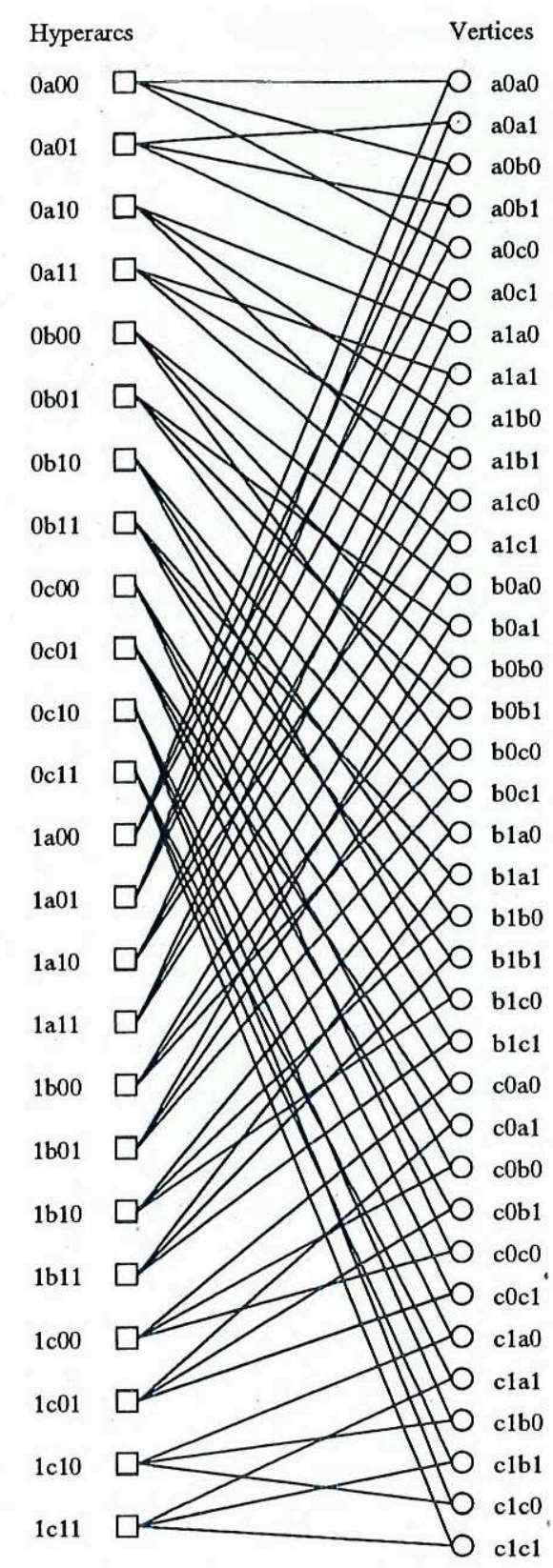

Connections from hyperarcs to vertices.

Note: All edges are directed from left to right.

Fig. 1. $R\left(B_{1}(2,2,3)\right)$ with $A=\{0,1\}$, and $B=\{a, b, c\}$.

$d(v+n)+\alpha=d v+d n+\alpha \equiv d v+\alpha \quad(\bmod m)$.

This is possible if and only if $d n \equiv 0(\bmod m)$. Similarly, we require that two integers congruent modulo $m$ represent the same hyperarc. Condition (2) satisfies this requirement.

We will now study the in-degree, in-size, and diameter of $G B_{2}(d, n, s, m)$.
Proposition 1. If $E$ is a hyperarc in $G B_{2}(d, n, s, m)$, then $\left|E^{-}\right|=d n / m=s^{-}$.

Proof. Every vertex $v$ is incident to $d$ hyperarcs numbered from $d v(\bmod m)$ to $d v+d-1(\bmod m)$ due to Condition (1). The set of $n$ vertices (whole vertex set) is incident to the hyperarcs numbered from 0 through $d n$ $-1(\bmod m)$. Thus, $\left|E^{-}\right|=d n / m=s^{-}$, for every hyperarc $E$, since $d n \equiv 0(\bmod m)$ by definition. 


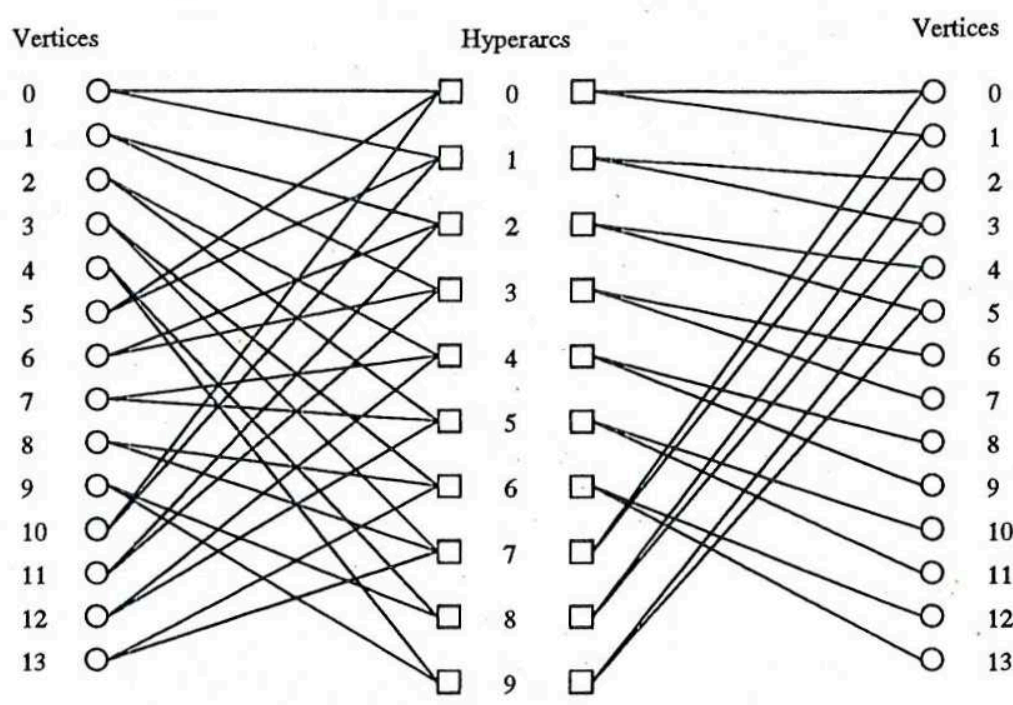

Connections from vertices to hyperarcs.

Connections from hyperarcs to vertices.

Note: All edges are directed from left to right.

Fig. 2.

Proposition 2. If $v$ is a vertex in $G B_{2}(d, n, s, m)$, then $d^{-}(v)=s m / n=d^{-}$.

Proof. Similar to the proof of Proposition 1.

Corollary 3. $\left|E^{-}\right|=\left|E^{+}\right|=s$ for every hyperarc $E$ and $d^{-}(v)=d^{+}(v)=d$ for every vertex $v$ in $G B_{2}(d, n$, $s, m)$ if and only if $d n=s m$.

Theorem 4. The underlying multidigraph of $G B_{2}(d, n$, $s, m)$ is $G B(d s, n)$.

Proof. When Conditions (1) and (2) are satisfied, vertex $v$ is joined via hyperarcs $E \equiv d v+\alpha(\bmod m)$ to the vertices $v^{\prime} \equiv(d s) v+\alpha s+\beta(\bmod n)$, for $0 \leq \alpha \leq d$ -1 , and $0 \leq \beta \leq s-1$. Indeed, $E=d v+\alpha+k m$ for some integer $k$, and

$$
\begin{aligned}
v^{\prime} & =s(d v+\alpha+k m)+\beta \\
& \equiv(d s) v+\alpha s+\beta \quad(\bmod n),
\end{aligned}
$$

which means that $v$ is adjacent to the vertices numbered $(d s) v+\gamma(\bmod n)$, for all $0 \leq \gamma \leq d s-1$. This is the same as the adjacency rule of $G B(d s, n)$.

Corollary 5. $D\left(G B_{2}(d, n, s, m)\right)=\left\lceil\log _{d s} n\right\rceil$.

If Conditions (1) and (2) are not satisfied, then Proposition 1, Proposition 2, and Theorem 4 do not hold. We will demonstrate this with an example: Consider Figure 2 where $n=14, m=10, d^{+}=2$, and $s^{+}=2$. Observe that the incidence relations comply with Rules (3) and
(4). However, we have $\left|E_{j}^{-}\right|=3$, for $0 \leq j \leq 7$, and $\left|E_{j}^{-}\right|=2$, for $j=8$, 9. Similarly, $d^{-}\left(v_{i}\right)=2$ for 0 $\leq i \leq 5$, and $d^{-}\left(v_{i}\right)=1$ for $6 \leq i \leq 13$. Observe also that vertices $v_{12}$ and $v_{13}$ cannot be reached in two steps starting from vertex $v_{6}$, and the diameter is $3>\left\lceil\log _{4} 14\right\rceil$ $=2$. This is due to the fact that the hyperarcs incident to vertex $n-1$ and vertex 0 are not consequently numbered ( similarly the vertices incident to the hyperarcs $m-1$ and 0 are not consequently numbered).

\subsection{Alphabetical Definition of Scheme 2}

If $n=(d s)^{D}$ and $m=d^{2}(d s)^{D-1}$, then we can give an alphabetical definition of the hypergraphs obtained using Rules (3) and (4). However, this definition is more complicated than that of Scheme 1.

Let $A$ and $B$ be two alphabets of sizes $d$ and $s$, respectively. Let the form of vertex labels be $[B A]^{D}$ and the form of hyperarc labels be $[A][B A]^{D-1}[A]$. We define the following bijections between vertex labels and integers modulo $n=(d s)^{D}$ and between the hyperarc labels and integers modulo $m=d^{2}(d s)^{D-1}$.

- Vertex label $\left(b_{1}, a_{1}, \ldots, b_{D-1}, a_{D-1}, b_{D}, a_{D}\right)$ represents the integer

$$
\sum_{i=1}^{D}\left(b_{i} d+a_{i}\right)(d s)^{D-t} \text {. }
$$

- Hyperarc label $\left(a_{0}, b_{1}, a_{1}, \ldots, b_{D-1}, a_{D-1}, a_{D}\right)$ represents the integer 


$$
a_{0} d(d s)^{D-1}+\sum_{i=1}^{D-1}\left(b_{i} d+a_{i}\right) d(d s)^{D-i-1}+a_{D}
$$

We now give the alphabetical equivalents of Rules (3) and (4). Without loss of generality, assume that the label of vertex $v$ is $\left(b_{1}, a_{1}, \ldots, b_{D-1}, a_{D-1}, b_{D}, a_{D}\right)$. By replacing $v$ with $\sum_{i=1}^{D}\left(b_{i} d+a_{i}\right)(d s)^{D-i}$ in Rule (3), we find that vertex $v$ is incident to the following hyperarcs:

$$
\begin{aligned}
d \sum_{i=1}^{D}\left(b_{i} d+a_{i}\right)(d s)^{D-i}+\alpha & \left(\bmod d^{2}(d s)^{D-1}\right), \quad 0 \leq \alpha \leq d-1 \\
\equiv a_{1} d(d s)^{D-1}+ & \sum_{i=2}^{D}\left(b_{i} d+a_{i}\right) d(d s)^{D-i}+\alpha \\
& \left(\bmod d^{2}(d s)^{D-1}\right), \quad 0 \leq \alpha \leq d-1 .
\end{aligned}
$$

Observe that this is the set of hyperarcs $\left\{\left(a_{1}, b_{2}, a_{2}, \ldots\right.\right.$, $\left.\left.b_{D}, a_{D}, \alpha\right) \mid \alpha \in A\right\}$. Therefore, the alphabetical equivalent of Rule (3) is that the vertex with label $\left(b_{1}, a_{1}, \ldots\right.$, $\left.b_{D-1}, a_{D-1}, b_{D}, a_{D}\right)$ is incident to the set of hyperarcs $\left\{\left(a_{1}, b_{2}, a_{2}, \ldots, b_{D}, a_{D}, \alpha\right) \mid \alpha \in A\right\}$.

To find the alphabetical equivalent of Rule (4), assume without loss of generality that the label of hyperarc $E$ is $\left(a_{0}, b_{1}, a_{1}, \ldots, b_{D-1}, a_{D-1}, a_{D}\right)$ and replace $E$ with $a_{0} d(d s)^{D-1}+\sum_{i=1}^{D-1}\left(b_{i} d+a_{i}\right) d(d s)^{D-i-1}+a_{D}$ in Rule (4). Then, the vertices in $E^{+}$are

$$
\begin{gathered}
s\left[a_{0} d(d s)^{D-1}+\sum_{i=1}^{D-1}\left(b_{i} d+a_{i}\right) d(d s)^{D-i-1}+a_{D}\right]+\beta \\
\left(\bmod (d s)^{D}\right), \quad 0 \leq \beta \leq s-1 \\
\equiv \sum_{i=1}^{D-1}\left(b_{i} d+a_{i}\right)(d s)^{D-i}+a_{D} s+\beta
\end{gathered}
$$$$
\left(\bmod (d s)^{D}\right), \quad 0 \leq \beta \leq s-1 .
$$

Therefore, the alphabetical equivalent of Rule (4) is that the out-set of the hyperarc $E$ with label $\left(a_{0}, b_{1}, a_{1}, \ldots\right.$, $\left.b_{D-1}, a_{D-1}, a_{D}\right)$ is

$$
\begin{aligned}
E^{+}=\left\{\left(b_{1}, a_{1}, \ldots, b_{D-1}, a_{D-1}, b_{x}, a_{x}\right)\right. & \\
& \left.b_{x} d+a_{x}=a_{D} s+\beta, 0 \leq \beta \leq s-1\right\} .
\end{aligned}
$$

As in the case of Scheme 1, each hyperarc in Scheme 2 corresponds to a subdiclique of a "natural diclique" of $B(d s, D)$. Natural dicliques of $B(d s, D)$ contain the arcs joining the vertices $\left(\beta, \alpha, b_{1}, a_{1}, \ldots, b_{D-1}, a_{D-1}\right)$ to $\left(b_{1}\right.$, $\left.a_{1}, \ldots, b_{D-1}, a_{D-1}, \beta^{\prime}, \alpha^{\prime}\right)$. They are partitioned into smaller dicliques by fixing $\alpha=a_{0}$ and letting $\beta^{\prime}, \alpha^{\prime}$ be such that $\beta^{\prime} d+\alpha^{\prime}=a_{D} s+\beta, 0 \leq \beta \leq s-1$. This corresponds to taking $s$ consecutive integers in the usual numbering of vertices.
These hypergraphs satisfy the condition $d n=s m$. Thus, they are uniform and regular and their diameter is $D$. We denote the networks of this special case by $B_{2}(d$, $D, s)$. Figure 3 depicts the bipartite digraph representation of $B_{2}(2,2,3)$.

\subsection{Nonisomorphism of Scheme 1 and Scheme 2}

Theorem 6. If $d, s \geq 2$, then $B_{1}(d, D, s)$ and $B_{2}(d, D$, $s)$ are not isomorphic.

Proof. Let $(a, b, a, b, a, \ldots, b, a, a)$ be a vertex in $R\left(B_{1}(d, D, s)\right)$ corresponding to a hyperarc of $B_{1}(d$, $D, s)$ for some $a \in A$ and $b \in B$. This vertex is on the following $s$ cycles of length $2 D$ (for all $\beta \in B$ ):

$$
\begin{array}{ccc}
(a, b, a, b, a, & \cdots & b, a, a) \\
(b, a, b, a & \cdots & b, a, \beta, a) \\
(a, b, a, b, a, & \cdots & \beta, a, a) \\
& \vdots & \\
(\beta, a, b, a & \cdots & b, a, b, a) \\
(a, b, a, b, a, & \cdots & b, a, a) .
\end{array}
$$

Now, let us look at the cycles of length $2 D$ in $R\left(B_{2}(d\right.$, $D, s)$ ). Let $E$ be a hyperarc in $B_{2}(d, D, s)$ and $e$ be its vertex image in $R\left(B_{2}(d, D, s)\right)$. The numbers corresponding to the vertices that are reachable from vertex $e$ in $2 D$ steps [i.e., the hyperarcs at distance $D$ from hyperarc $E$ in $\left.B_{2}(d, D, s)\right]$ form a set of $(d s)^{D}$ consecutive integers. Thus, there are at most

$$
\left\lceil\frac{(d s)^{D}}{d^{2}(d s)^{D-1}}\right\rceil=\left\lceil\frac{s}{d}\right\rceil
$$

cycles of length $2 D$, containing the vertex $e$. The theorem follows, since $\lceil s / d\rceil<s$, for $d>1$.

Another way to prove Theorem 6 is to compare the duals of $B_{1}(d, D, s)$ and $B_{2}(d, D, s)$. Observe that the dual of $B_{2}(d, D, s)$ is also a de Bruijn hypergraph, namely, $B_{2}(s, D, d)$, i.e., de Bruijn hypergraph of Scheme 2 with out-degree $s$, out-size $d$, and diameter $D$, whereas the diameter of the dual of $B_{1}(d, D, s)$ is $D+1$.

\subsection{Arithmetical Definition of Scheme 1}

It is also possible to generalize the hypergraphs of Scheme 1 for any number of vertices using arithmetic congruences. Let $d$ be the vertex out-degree and $n$ be the number 
of vertices. Choose the number of hyperarcs $m$ and the hyperarc out-size $s$ such that

$$
\begin{aligned}
& d n \equiv 0 \quad(\bmod m) \\
& s m \equiv 0 \quad(\bmod n) \\
& m \equiv 0 \quad(\bmod d) \text {. }
\end{aligned}
$$

Assume that the vertices are numbered with integers modulo $n$ and the hyperarcs are numbered with integers modulo $m$. Then, the incidence relations between vertices and hyperarcs are as follows: Vertex $v$ is incident to the hyperarcs

$$
\begin{aligned}
E \equiv d v+\alpha \quad(\bmod m), & \\
& \text { for all } 0 \leq \alpha \leq d-1,
\end{aligned}
$$

and hyperarc $E$ is incident to the vertices

$$
u \equiv s\left(\left\lfloor\frac{E}{d}\right\rfloor d\right)+\beta d+\left(E-\left\lfloor\frac{E}{d}\right\rfloor d\right)
$$

$$
(\bmod n), \text { for all } 0 \leq \beta \leq s-1 \text {. }
$$

Let $G B_{1}(d, n, s, m)$ denote the hypergraphs constructed using Rules (7) and (8) above. Note that if $s$ $=1$ we obtain the generalized de Bruijn digraphs. We will now study the in-degree, in-size, and diameter of $G B_{1}(d, n, s, m)$.

Proposition 7. If $E$ is a hyperarc in $G B_{1}(d, n, s, m)$, then $\left|E^{-}\right|=d n / m=s^{-}$.

Proof. Same as the proof of Proposition 1.

Proposition 8. If $v$ is a vertex in $G B_{1}(d, n, s, m)$, then $d^{-}(v)=s m / n=d^{-}$.

Proof. Let $E$ be a hyperarc and $u$ be the vertex with number $u=\lfloor E / d\rfloor(d s)+E-\lfloor E / d\rfloor d(\bmod n)$. Then, $E$ is incident to the $s$ vertices with numbers $u+\beta d(\bmod$ $n$ ) for all $0 \leq \beta \leq s-1$. A cluster of $d$ hyperarcs, numbered consecutively from $\lfloor E / d\rfloor d$ to $\lfloor E / d\rfloor d+d$ -1 , are incident to $d s$ vertices, numbered consecutively from $\lfloor E / d\rfloor d s(\bmod n)$ to $\lfloor E / d\rfloor d s+d s-1(\bmod n)$. Since $m$ is chosen to be a multiple of $d, m$ hyperarcs are incident to $s m$ vertices, numbered consecutively from 0 to $s m-1(\bmod n)$. Every vertex is in the in-set of $d^{-}$ $=s m / n$ hyperarcs, because $s m \equiv 0(\bmod n)$.
We will demonstrate with an example (Fig. 4) that if $m$ is not a multiple of $d$ then Proposition 8 does not hold. Let $n=10$ and $d=3$. Choose $m=10$ and $s=2$. The in-degrees of vertices $v_{1}$ and $v_{9}$ are $d^{-}\left(v_{1}\right)=3$, and $d^{-}\left(v_{9}\right)$ $=1$.

Theorem 9. The underlying multidigraph of $G B_{1}(d, n$, $s, m)$ is $G B(d s, n)$.

Proof. Let $v$ be a vertex such that $v \in E^{-}$. We can write $E=d v+\alpha+k m$ where $k$ is an integer and 0 $\leq \alpha \leq d-1$. The condition $m \equiv 0(\bmod d)$ implies that $\lfloor E / d\rfloor d=d v+k m$, and the condition $s m \equiv 0(\bmod n)$ implies that $u \equiv d s v+\beta d+\alpha(\bmod n)$. Therefore, $v$ is adjacent to the vertices $d s v+\gamma, 0 \leq \gamma \leq d s-1$, which is exactly same as the adjacency rule in $G B(d s, n)$.

Corollary 10. $D\left(G B_{1}(d, n, s, m)\right)=\left\lceil\log _{d s} n\right\rceil$.

Theorem 11. If $n=(d s)^{D}$ and $m=d^{2}(d s)^{D-1}$, then $G_{1}(d, n, s, m)$ is isomorphic to $B_{1}(d, D, s)$.

Proof. We will use Bijection (5), defined in Section 3.5 between vertex labels and integers modulo $(d s)^{D}$ and Bijection (6) between hyperarc labels and integers modulo $d^{2}(d s)^{D-1}$. Then,

$$
E-\left\lfloor\frac{E}{d}\right\rfloor d=a_{D}
$$

and

$$
s\left\lfloor\frac{E}{d}\right\rfloor d \quad\left(\bmod (d s)^{D}\right)=\sum_{i=1}^{D-1}\left(b_{i} d+a_{i}\right)(d s)^{D-i} ;
$$

therefore, the vertices in $E^{+}$are $\sum_{i=1}^{D-1}\left(b_{i} d+a_{i}\right)(d s)^{D-i}$ $+\beta d+a_{D}$, which corresponds to the vertices with labels $\left(b_{1}, a_{1} \cdots, b_{D-1}, a_{D-1}, \beta, a_{D}\right)$, where $0 \leq \beta \leq s-1$.

\section{KAUTZ BUS NETWORKS}

\subsection{Kautz Digraphs}

An important class of digraphs with large number of vertices and small diameter is the Kautz digraphs [15]. One of the definitions of the Kautz digraphs is based on alphabets: Let $d$ and $D$ be two positive integers and $A$ be an alphabet of $d+1$ letters. The vertices of Kautz digraph $K(d, D)$ are labeled by strings of $D$ letters from $A$, such that no two consecutive letters are identical. Vertex $\left(a_{1}\right.$, $\left.a_{2}, \ldots, a_{D}\right)$ is joined to the vertices $\left(a_{2}, \ldots, a_{D}, \alpha\right)$ for all $\alpha \in A-\left\{a_{D}\right\}$. It is easy to see that $K(d, D)$ is a regular digraph with in- and out-degree $d$. It has $d^{D}$ $+d^{D-1}$ vertices and its diameter is $D$.

A class of digraphs that generalize Kautz digraphs was 


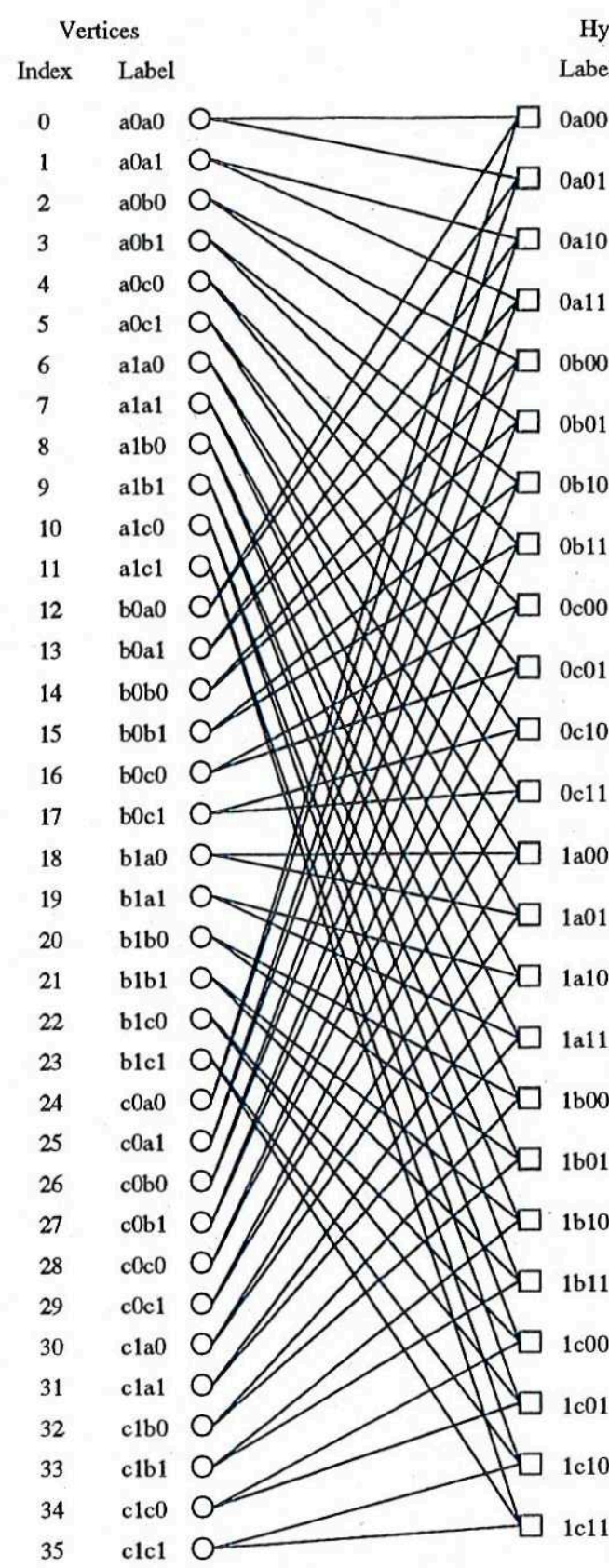

Hyperarcs

Vertices

Label Index

Label Index

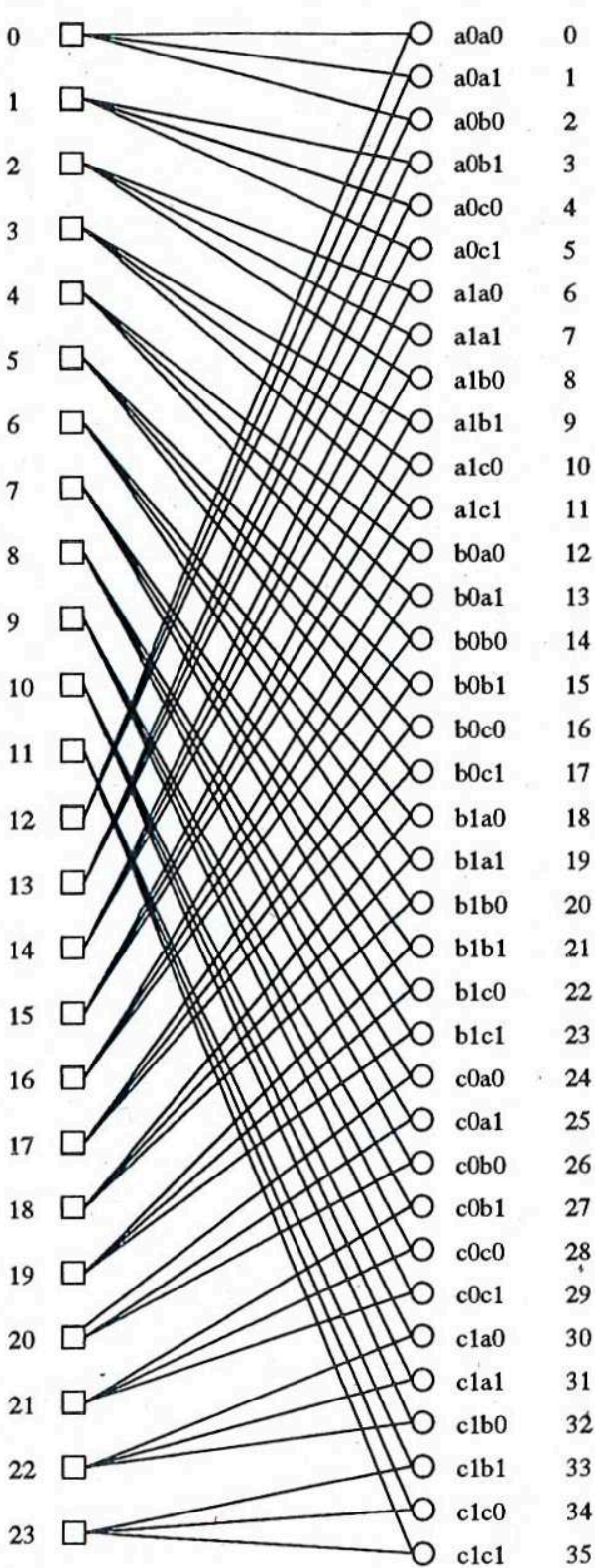

Connections from vertices to hyperarcs.

Connections from hyperarcs to vertices.

Note: All edges are directed from left to right.

Fig. 3. $R\left(B_{2}(2,2,3)\right)$ with $A=\{0,1\}$, and $B=\{a, b, c\}$.

defined by Imase and Itoh [14] using arithmetic congruences: The vertices are numbered with integers modulo $n$. If the out-degree is $d$, then vertex $v$ is joined to vertices $u \equiv-d v-\alpha(\bmod n)$, for $1 \leq \alpha \leq d$. The diameter of the resulting digraph is at $\operatorname{most}\left\lceil\log _{d} n\right\rceil$. Furthermore, if $n=d^{D}+d^{D-k}$ for a positive odd integer $k$, then the diameter is $D$. If $n=d^{D}+d^{D-1}$, these digraphs are isomorphic to $K(d, D)$. Therefore, we call these digraphs the generalized Kautz digraphs and denote them by $G K(d, n)$.

\subsection{Arithmetical Definition}

In this section, we define a family of directed hypergraphs using arithmetic congruences. Let $n$ be the number of 


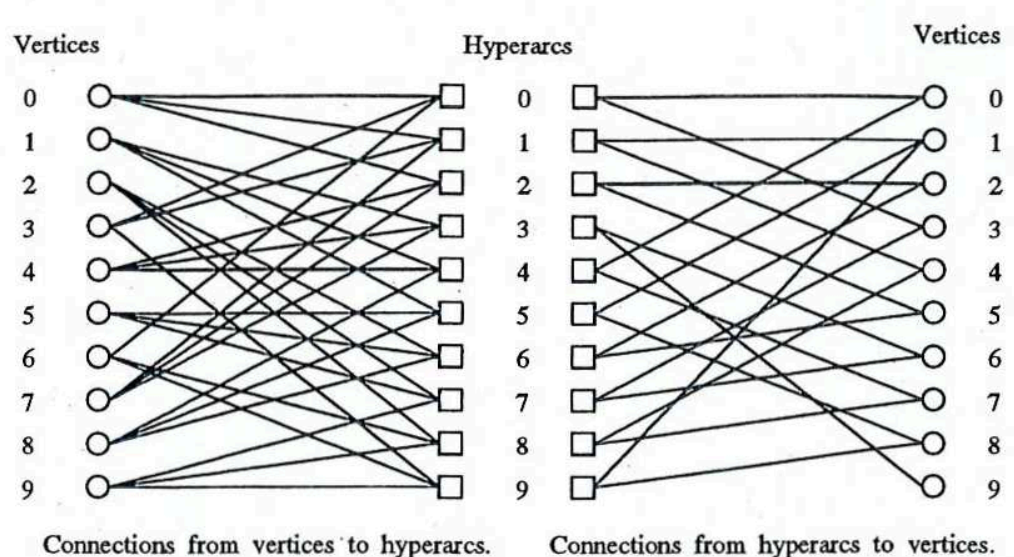

Note: All edges are directed from left to right.

Fig. 4.

vertices and $d$ be the vertex out-degree. Choose the number of hyperarcs $m$ and the hyperarc out-size $s$ such that

$$
\begin{array}{ll}
d n \equiv 0 & (\bmod m) \\
s m \equiv 0 & (\bmod n) .
\end{array}
$$

Assume that the vertices are numbered with integers modulo $n$ and that the hyperarcs are numbered with integers modulo $m$. The incidence rules are as follows: Vertex $v$ is incident to the hyperarcs

$$
E \equiv d v+\alpha \quad(\bmod m), \quad 0 \leq \alpha \leq d-1,
$$

and the out-set of the hyperarc $E$ consists of the vertices

$$
u \equiv-s E-\beta \quad(\bmod n), \quad 1 \leq \beta \leq s .
$$

We call these hypergraphs the generalized Kautz hypergraphs since, as we will see later, their underlying multidigraphs are the generalized Kautz digraphs. We denote them by $G K(d, n, s, m)$. Figure 5 depicts the bipartite representation digraph of $G K(2,42,3,28)$. In the case $d=s$, bipartite digraphs using the same rules were also constructed by Fiol and Yebra [11], in order to obtain large bipartite digraphs.

Proposition 12. The following incidence rules can alternatively be used in place of Rules (9) and (10), respectively, in the definition of $G K(d, n, s, m)$ :

Vertex $v$ is incident to the hyperarcs

$$
E \equiv-d v-\alpha \quad(\bmod m) \quad 1 \leq \alpha \leq d,
$$

and the out-set of hyperarc E consists of the vertices

$$
u \equiv s E+\beta \quad(\bmod n) \quad 0 \leq \beta \leq s-1 .
$$

Proof. Define a bijection $\phi$ on the set of integers modulo $m$ with $\phi(i)=-i-1$. Let $\phi^{-1}$ denote the inverse function of $\phi$. Observe that $\phi=\phi^{-1}$. Using the bijection $\phi$, renumber the hyperarcs of $G K(d, n, s, m)$.

To preserve the incidence relation defined in Rule (9), vertex $v$ must be incident to the set of hyperarcs

$$
\begin{aligned}
& \{\phi(d v+\alpha) \quad(\bmod m) \mid 0 \leq \alpha \leq d-1\} \\
\equiv & \{-d v-\alpha-1 \quad(\bmod m) \mid 0 \leq \alpha \leq d-1\} \\
\equiv & \{-d v-\alpha \quad(\bmod m) \mid 1 \leq \alpha \leq d\} .
\end{aligned}
$$

The out-set of hyperarc $E$ is, by Rule (10),

$$
\begin{aligned}
& \left\{-s \cdot \phi^{-1}(E)-\beta \quad(\bmod n) \mid 1 \leq \beta \leq s\right\} \\
\equiv & \{-s \cdot(-E-1)-\beta \quad(\bmod n) \mid 1 \leq \beta \leq s\} \\
\equiv & \{s E+(s-\beta) \quad(\bmod n) \mid 1 \leq \beta \leq s\} \\
\equiv & \{s E+\beta \quad(\bmod n) \mid 0 \leq \beta \leq s-1\} .
\end{aligned}
$$

Hence, we obtain Rule (11) and Rule (12).

Figure 6 depicts the bipartite representation digraph of $G K(2,42,3,28)$, constructed using Rules (11) and (12).

\subsection{Properties of Kautz Bus Networks}

Proposition 13. If $E$ is a hyperarc in $G K(d, n, s, m)$, then $\left|E^{-}\right|=d n / m=s^{-}$.

Proof. Every vertex $v$ is incident to the $d$ hyperarcs numbered from $d v(\bmod m)$ to $d v+d-1(\bmod m)$. The set of $n$ vertices (whole vertex set) is incident to the hyperarcs numbered from 0 through $d n-1(\bmod m)$. 


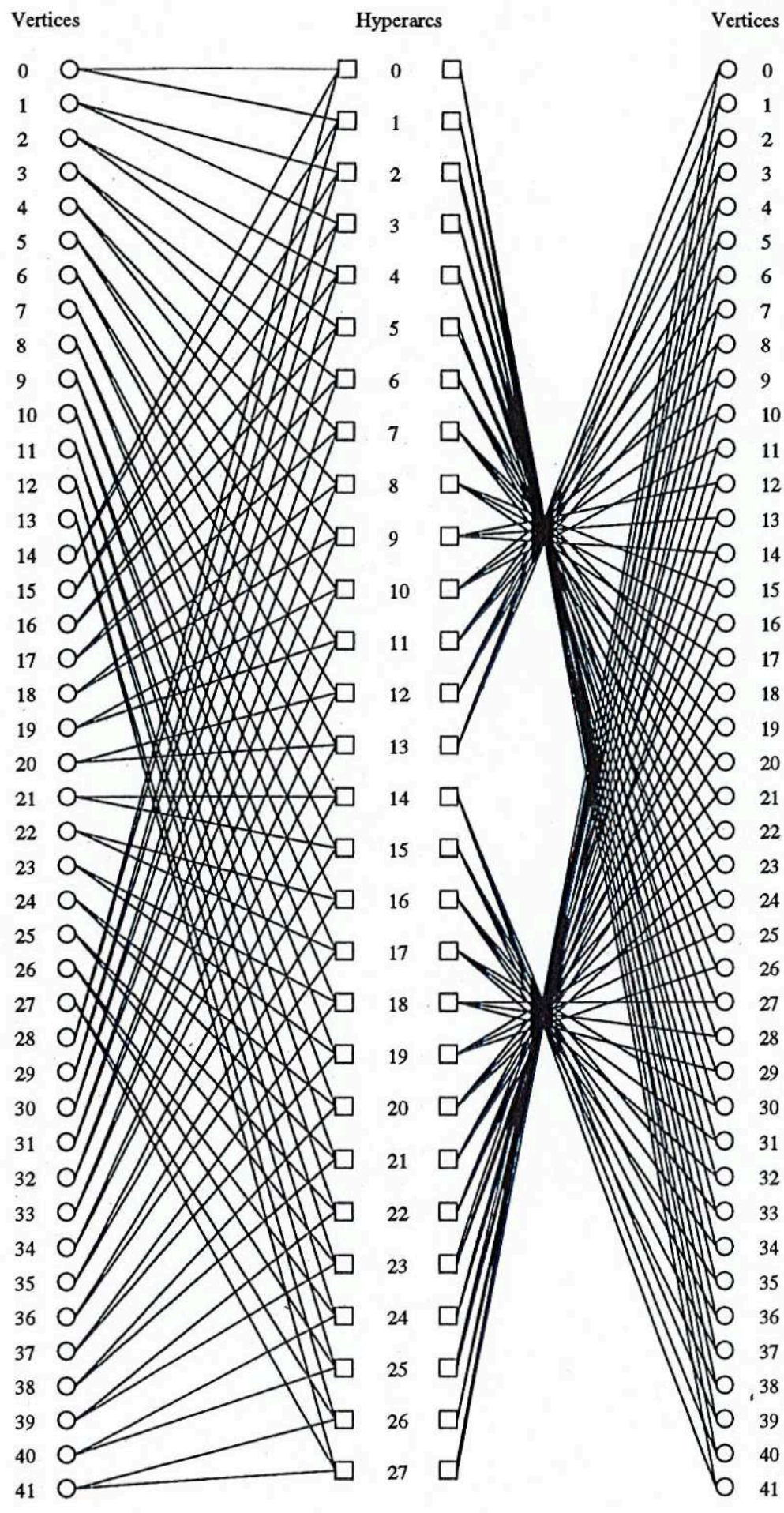

Connections from vertices to hyperarcs Connections from hyperarcs to vertices.

Note: All edges are directed from left to right.

Fig. 5. The bipartite representation digraph of $\operatorname{GK}(2,42,3,28)$. 


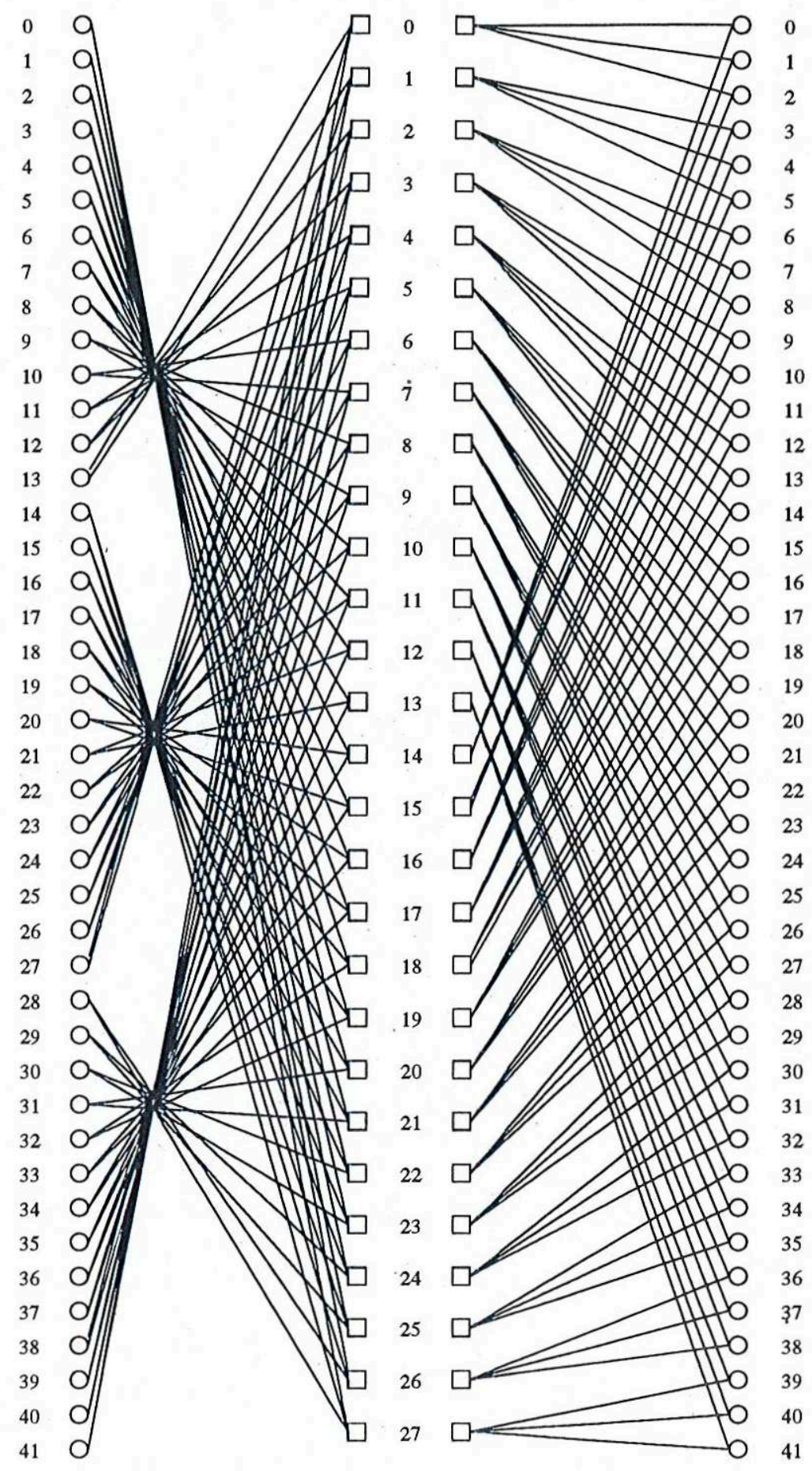

Connections from vertices to hyperarcs.

Connections from hyperarcs to vertices.

Note: All edges are directed from left to right.

Fig. 6. The bipartite representation digraph of $\operatorname{GK}(2,42,3,28)$. 
Since $d n \equiv 0(\bmod m)$ by definition, every hyperarc has an equal number of vertices, $s^{-}=d n / m$, in its in-set.

Proposition 14. If $v$ is a vertex in $G K(d, n, s, m)$, then $d^{-}(v)=s m / n=d^{-}$.

Proof. Similar to the proof of Proposition 13.

Corollary 15. $\left|E^{-}\right|=\left|E^{+}\right|=s$ for every hyperarc $E$ and $d^{-}(v)=d^{+}(v)=d$ for every vertex $v$ in $G K(d ; n$, $s, m)$ if and only if $d n=s m$.

Theorem 16. The underlying multidigraph of $G K(d, n$, $s, m)$ is $G K(d s, n)$.

Proof. In $G K(d, n, s, m)$, the vertex $v$ is adjacent to the vertices $(0 \leq \alpha \leq d-1,1 \leq \beta \leq s)$ :

$$
\begin{aligned}
u & \equiv-s((d v+\alpha)(\bmod m))-\beta(\bmod n), \\
& \equiv-s(d v+\alpha-k m)-\beta \quad(\bmod n),
\end{aligned}
$$

for some $k \geq 0$,

$$
\begin{aligned}
& \equiv-d s v-s \alpha+k s m-\beta \quad(\bmod n), \\
& \equiv-d s v-(s \alpha+\beta) \quad(\bmod n) \\
& \equiv-d s v-\gamma \quad(\bmod n), \quad 1 \leq \gamma \leq d s,
\end{aligned}
$$

which are exactly the same as the neighbours of $v$ in $G K(d s, n)$.

Corollary 17. Diameter of $G K(d, n, s, m)$ is at most $\left\lceil\log _{d s} n\right\rceil$. Furthermore, the diameter is $D$, if $n=(d s)^{D}$ $+(d s)^{D-k}$, where $k$ is a positive odd integer.

Corollary 18. $G K(d, d s+1, s, m)$ is a directed Moore hypergraph of diameter one if there exist positive integers $d, s, m$ such that

$$
\begin{aligned}
d^{2} s+d \equiv 0 & & (\bmod m) \\
s m \equiv 0 & & (\bmod d s+1) .
\end{aligned}
$$

\section{CONCLUSION}

We have defined two schemes to obtain bus networks from de Bruijn digraphs and one scheme to obtain bus networks from Kautz digraphs.

We showed that the directed de Bruijn bus networks of maximum out-degree $d$, maximum bus out-size $s$, and diameter $D$ have $(d s)^{D}$ processors. Also, we presented generalizations of the directed de Bruijn bus networks for arbitrary numbers of processors and established that their diameter is logarithmic in terms of the number of proces- sors. Similar properties hold for the directed Kautz bus networks which connect $(d s)^{D}+(d s)^{D-1}$ processors.

We can easily obtain undirected bus networks from the aforementioned networks, by ignoring the orientation of the buses. Undirected de Bruijn bus networks have $((\Delta r) / 4)^{D}$ processors where $\Delta$ is the maximum degree and $r$ is the maximum bus size. Undirected Kautz bus networks, on the other hand, connect $((\Delta r) / 4)^{D}+((\Delta r) / 4)^{D-1}$ processors.

We can, furthermore, obtain bus networks on any number of processors and with logarithmic diameter, by ignoring the orientation of the buses of the generalized de Bruijn or Kautz bus networks.

The connectivity, network load, and routing issues for both directed and undirected de Bruijn and Kautz bus networks are being considered in [3] and ongoing research. In [4], we show that definitions of the de Bruijn digraphs and of the Kautz digraphs using line digraph iterations can also be extended to hypergraphs by using the "directed line hypergraph" technique.

F. Ö. E. would like to thank Dr. Johny Bond and Dr. Patrick Solé for valuable discussions. J.-C. B. was supported by the French GDR-PRC C ${ }^{3}$; R. W. Dawes, by the Natural Science and Engineering Research Council of Canada; and F.Ö.E., by the School of Graduate Studies and Research, Queen's University at Kingston.

\section{REFERENCES}

[1] J.-C. Bermond, J. Bond, M. Paoli, and C. Peyrat, Graphs and interconnection networks: Diameter and vulnerability. Surveys in Combinatorics, Invited Papers for the Ninth British Combinatorial Conference (E. K. Lloyd, Ed.), Vol. 82, London Mathematics Society Lecture Note Series. Cambridge University Press, Cambridge (1983) $1-30$.

[2] J.-C. Bermond, C. Delorme, and J.-J. Quisquater, Strategies for interconnection networks: Some methods from graph theory. J. Parallel Distrib. Process. 3 (1986) 433-449.

[3] J.-C. Bermond and F. Ö. Ergincan, Connectivity in bus interconnection networks. In preparation.

[4] J.-C. Bermond and F. Ö. Érgincan, Directed line hypergraphs. In preparation.

[5] J.-C. Bermond and F. Ö. Ergincan, Bus interconnection networks. Technical Report 93-56, I3S, CNRS, Université de Nice-Sophia Antipolis, France (1993) Discr. Appl. Math. 68 (1996) 1-15.

[6] J.-C. Bermond, N. Homobono, and C. Peyrat, Large fault-tolerant interconnection networks. Graphs Combin. 5 (1989) 107-123.

[7] J.-C. Bermond and C. Peyrat, The de Bruijn and Kautz networks: A competitor for the hypercube? Hypercube and Distributed Computers, Proceedings of the First European Workshop on Hypercube and Distributed Com- 
puters, Rennes (Oct. 1989) Elsevier, Amsterdam (North-Holland) (1989) 279-293.

[8] F. R. K. Chung, Diameters of communication networks. AMS Proceedings of Symposia in Applied Mathematics 34 (1986) 1-18.

[9] K. Doty, Dense bus connection networks. Proceedings of the 1983 International Conference on on Parallel Processing (1983) 158-160.

[10] F. Ö. Ergincan and D. A. Gregory, Directed Moore hypergraphs. Discr. Appl. Math. 63 (1995) 117-127.

[11] M. A. Fiol and J. L. A. Yebra, Dense bipartite digraphs. J. Graph Theory 14 (1990) 687-700.

[12] M. A. Fiol, J. L. A. Yebra, and I. A. de Miquel, Line digraph iterations and the $(d, k)$ digraph problem. IEEE Trans. Comput. C-33 (1984) 400-403.

[13] M. Imase and M. Itoh, Design to minimize diameter on building-block network. IEEE Trans. Comput. C-30 (1981) 439-442.

[14] M. Imase and M. Itoh, A design for directed graphs with minimum diameter. IEEE Trans. Comput. C-32 (1983) $782-784$.

[15] W. H. Kautz, The design of optimum interconnection networks for multiprocessors. Architecture and Design of Digital Computer, NATO Advanced Summer Institute, (1969) 249-277.

[16] S. M. Reddy, D. K. Pradhan, and J. G. Kuhl, Directed graphs with minimal diameter and maximal connectivity. Technical Report, Oakland University, School of Engineering (1980).

[17] M. R. Samatham and D. K. Pradhan, The de Bruijn multiprocessor network: A versatile parallel processing and sorting network for VLSI. IEEE Trans. Comput. 38 (1989) 567-581 (corrections in IEEE Trans. Comput. 40 (1991) 122-123). 